\title{
Uso de bebidas alcoólicas em postos de gasolina de Porto Alegre: estudo piloto
}

\author{
Alcohol use on premises of gas stations of Porto Alegre, Brazil: pilot study
}

\author{
Raquel De Boni ${ }^{1}$, Daniela Benzano ${ }^{2}$, Carl Leukefeld ${ }^{3}$, Flavio Pechansky ${ }^{4}$ \\ ${ }^{1}$ Médica psiquiatra. Mestrando. ${ }^{2}$ Estatística. ${ }^{3}$ Doutor. Diretor, Center on Drug and Alcohol Research, Universidade de Kentucky, Lexington, EUA. \\ ${ }^{4}$ Doutor. Diretor, Centro de Pesquisa em Álcool e Drogas, Universidade Federal do Rio Grande do Sul (UFRGS), Porto Alegre, RS.
}

\section{Resumo}

Introdução: O uso de álcool é um forte preditor de acidentes de trânsito. Este artigo descreve a factibilidade e dados preliminares de um estudo que avalia a alcoolemia entre jovens que utilizam bebidas alcoólicas em estacionamentos de postos de gasolina de Porto Alegre (RS).

Método: Estudo transversal, com amostra em alvo, estratificada por idade e gênero. Os dados foram coletados através de um questionário autopreenchido, e a alcoolemia foi estimada com uso de bafômetro.

Resultados: A taxa de recusa foi de 13,7\%, e medida da alcoolemia acima de 0,06\% foi encontrada em 35,5\% (IC95\% 24,3-47,9) da amostra. Aproximadamente $10 \%$ destes indivíduos (18\% dos homens) relatavam intenção de dirigir nas 2 horas subseqüentes à coleta. Conclusão: É discutida a potencial utilização deste tipo de dado, bem como a necessidade de novos estudos sobre uso de substâncias em motoristas brasileiros.

Descritores: Consumo de bebidas alcoólicas, acidentes de trânsito, assunção de riscos.

\begin{abstract}
Introduction: Alcohol use is a strong predictor of traffic accidents. This paper reports feasibility issues and preliminary data on blood alcohol concentration in youths who drink alcohol in gas stations of Porto Alegre, Brazil.

Method: It used a cross-sectional design with a purposive sample stratified by age and gender. Data were collected through a selfadministered questionnaire and blood alcohol concentration was estimated with breath alcohol analyzers.

Results: Refusal rate was $13.7 \%$ and blood alcohol concentration over $0.06 \%$ was found in $35.5 \%$ (95\%CI $24.3-47.9)$ of the sample. Almost $10 \%$ of the subjects (18\% of the males) reported they would drive within 2 hours of the interview.

Conclusion: The potential utilization of such measures is discussed, as well as the need for studies on substance use in young Brazilian drivers.
\end{abstract}

Keywords: Alcohol drinking, traffic accidents, risk-taking behavior.

\section{Introdução}

Segundo a Organização Mundial da Saúde, 1,2 milhão de pessoas morrem anualmente em conseqüência de acidentes de trânsito ${ }^{1}$. A maioria desses óbitos $(90 \%)$ e $91,8 \%$ dos 38 milhões de anos de vida perdidos ajustados por incapacitação (DALY) ocorrem em países em desenvolvimento ${ }^{1,2}$. O uso de álcool é um forte preditor de acidentes de trânsito, e existe uma correlação linear entre aumento de alcoolemia e risco de acidentes de trânsito ${ }^{3-5}$. O consumo excessivo de álcool - binge drinking (mais de cinco doses em uma única ocasião) -, o gênero e a idade estão fortemente associados a lesões causadas por acidentes de trânsito ${ }^{6,7}$.

Em 2004, foram reportados 35.674 óbitos secundários a acidentes de trânsito no Brasil ${ }^{8}$. Mesmo com números

\section{Correspondência:}

Centro de Pesquisa em Álcool e Drogas, UFRGS, Rua Ramiro Barcelos, 2350/2201-F, CEP 90035-903, Porto Alegre, RS. Tel.: (51) 3330.5813. Email: raqueldeboni@msn.com

Apoio financeiro: Fundação de Incentivo a Pesquisa (FIPE) do Hospital de Clínicas de Porto Alegre e Universidade de Kentucky. 
dessa magnitude, poucos estudos relatam a associação entre uso de álcool e acidentes de trânsito em motoristas e passageiros brasileiros ${ }^{9,10}$. Sabe-se que o uso de álcool está aumentando no Brasil ${ }^{11}$; e Porto Alegre (RS) apresenta uma alta prevalência desse uso entre os jovens, assim como uma das mais altas taxas de uso de maconha do país ${ }^{12}$. Uma prática comum entre adolescentes e adultos jovens na cidade é dirigir até lojas de conveniência de postos de gasolina, usualmente com amigos, e lá fazer uso de bebidas alcoólicas. Esses ambientes são bem iluminados e contam com seguranças particulares, razões pelas quais este comportamento poderia ser explicado. Não há estimativas dos níveis de alcoolemia ou das prevalências de outros fatores de risco para acidentes de trânsito associados com consumo de álcool em tais grupos, e também não há estudos sobre a percepção de risco para dirigir sob influência do álcool nesses jovens.

O artigo tem como objetivo descrever a factibilidade e os dados preliminares relacionados ao beber e dirigir entre jovens que consomem bebidas alcoólicas em postos de gasolina de Porto Alegre. Trata-se de um estudo piloto, que se insere em um estudo nacional sobre uso de álcool e drogas no trânsito brasileiro, financiado pela Secretaria Nacional Anti-Drogas (SENAD).

\section{Métodos}

Foi utilizado um delineamento transversal com amostra em alvo. A amostra foi obtida em quatro postos de gasolina de Porto Alegre. A seleção dos locais englobou as oito regiões da Secretaria de Saúde de Porto Alegre. Em cada região, dois postos de gasolina foram selecionados: um foi selecionado aleatoriamente, e o outro foi pareado após indicação do sindicato de proprietários de postos de gasolina. Dentro de cada região, os postos foram previamente visitados, e aquele com maior número de pessoas, nos horários estabelecidos para a coleta de dados, era escolhido. Os proprietários dos postos de gasolina foram contatados para obtenção de autorização para a coleta de dados, sendo que nenhum deles recusou.

\section{Amostragem}

A amostra foi estratificada por idade (menores e maiores de 21 anos) e por gênero. A coleta de dados foi realizada nos dias e horários descritos na literatura como sendo os que apresentam as maiores taxas de acidentes fatais secundários ao uso de álcool: sextas-feiras e sábados, das $23 \mathrm{~h}$ às $4 \mathrm{~h}^{13}$. Todos os indivíduos foram entrevistados em maio de 2006. A pesquisa foi realizada em dois postos de gasolina a cada noite, simultaneamente: das $23 \mathrm{~h}$ às $24 \mathrm{~h}$ e das $3 \mathrm{~h}$ às $4 \mathrm{~h}$. Em cada bloco de horário, dois homens e duas mulheres, acima e abaixo de 21 anos, eram entrevistados, totalizando 16 casos por noite. Os entrevistadores abordaram a primeira pessoa vista consumindo bebidas alcoólicas ao entrarem no posto de gasolina e, a seguir, a segunda pessoa, até que obtivessem quatro entrevistas por hora.

\section{Entrevistadores}

Dez entrevistadores foram treinados: dois psicólogos, um médico residente em psiquiatria e sete estudantes de medicina. Foram selecionados entrevistadores com idade e escolaridade semelhante à dos sujeitos de pesquisa. Eles saíam em duplas (sempre homem e mulher), vestiam camisetas identificando o estudo, apresentavam-se aos seguranças do posto de gasolina antes de entrevistar os indivíduos e abordavam indivíduos do sexo oposto. Esses procedimentos foram adotados com a intenção de reduzir as taxas de recusa.

\section{Critérios de inclusão}

Os critérios de inclusão foram: 1) ter, no mínimo, 15 anos; 2) estar consumindo bebida alcoólica; e 3 ) ser motorista ou passageiro de um veículo automotor.

\section{Medidas}

As informações sobre dados demográficos, uso de substâncias e comportamentos citados na literatura internacional como de risco para acidentes de trânsito relacionados ao uso de álcool (acidente prévio por beber e dirigir, ser passageiro de motorista intoxicado e uso de maconha) ${ }^{14,15}$ foram obtidas através de um questionário auto-aplicável, preenchido em cerca de 5 minutos. .

A alcoolemia foi estimada pela concentração de álcool no ar expirado, medida através de dois bafômetros convencionais (ALCO SENSOR e ALCO-SENSOR IV, Intoximeters, Inc.) previamente calibrados. Os entrevistadores receberam treinamento sobre sua utilização. A medida foi obtida após o preenchimento do questionário, quando os indivíduos deveriam ter permanecido ao menos 15 minutos sem beber ou fumar. Esse procedimento buscou estimar a alcoolemia precisa, e não apenas o álcool presente na cavidade oral.

\section{Consentimento informado}

Os aspectos éticos foram discutidos com o Comitê de Ética em Pesquisa do Hospital de Clínicas de Porto Alegre. Algumas das resoluções são descritas a seguir. Nenhuma informação que pudesse identificar os indivíduos foi coletada, garantindo anonimato em relação a potenciais questões legais. Aos motoristas com alcoolemia acima do limite permitido $(0,06 \%$ no Brasil $)$, foi solicitado que algum amigo que não estivesse bebendo dirigisse. Caso isso não fosse possível, foi 
oferecido transporte alternativo (táxi) através de um acordo prévio com uma empresa local. Cada potencial entrevistado recebeu explicações sobre o propósito do estudo e a confidencialidade dos dados, e assinou um termo de consentimento livre e esclarecido no caso de aceitar participar do estudo.

\section{Análise dos dados}

Os dados foram analisados usando o programa SPSS 14.0. As comparações de gênero foram realizadas através de testes do qui-quadrado para variáveis categóricas, teste $t$ de Student para variáveis contínuas simétricas, e teste U de Mann-Whitney para variáveis contínuas assimétricas. $\mathrm{O}$ nível de significância foi estabelecido em 0,05 .

\section{Resultados}

Setenta e três potenciais participantes foram abordados pelos entrevistadores. Dez $(13,7 \%)$ recusaramse em participar; não houve diferença significativa entre recusas por parte de homens e mulheres. Um sujeito foi excluído por inconsistência de dados no questionário. Não houve diferenças significativas nas características demográficas de homens e mulheres, exceto em relação à renda: $60 \%$ dos homens tinham renda maior de cinco salários-mínimos e $54 \%$ das mulheres estavam na faixa de um a cinco salários-mínimos $(\mathrm{p}<0.006)$. Aidade média foi 22,7 $( \pm 5,0)$, o número de anos de estudo foi $13( \pm 3,0$ anos), 75,8\% estavam trabalhando e $43,5 \%$ tinham parceiro fixo.

Alcoolemia maior de $0,06 \%$ foi encontrada em 35,5\% (IC95\% 24,3-47,9) da amostra. Cerca de 10\% desses indivíduos ( $18 \%$ dos homens) relataram que dirigiriam dentro de 2 horas após a entrevista, como pode ser observado na Tabela 1. Nenhum deles solicitou que um amigo dirigisse ou aceitou um táxi.

Não houve diferenças significativas quanto a comportamentos de risco de acidentes de trânsito entre jovens abaixo e acima de 21 anos. Oito indivíduos apresentavam menos de 18 anos (12,9\%) - idade legal para comprar bebidas alcoólicas no Brasil: sete deles comparam bebidas no posto de gasolina, e nenhum pretendia dirigir nas 2 horas seguintes à entrevista.

Tabela 1 - Comportamentos de risco para acidentes de trânsito estratificados por gênero

\begin{tabular}{|c|c|c|c|c|}
\hline & $\begin{array}{c}\text { Homens } \\
\mathrm{n}=\mathbf{3 2}(\%)\end{array}$ & $\begin{array}{c}\text { Mulheres } \\
\mathbf{n}=\mathbf{3 0}(\%)\end{array}$ & $\begin{array}{c}\text { Global } \\
\mathrm{n}=62(\%)\end{array}$ & OR (IC95\%) \\
\hline Alcoolemia (g/100 mL) & $0,05(0,0-0,21)^{*}$ & $0,02(0,0-0,16)^{*}$ & $0,03(0,0-0,21)^{*}$ & $1,67(0,92-2,9)^{\dagger}$ \\
\hline Alcoolemia $>0,06 \%$ & $15(46,9)$ & $7(23,3)$ & $22(35,5)$ & $2,89(0,97-8,69)$ \\
\hline $\begin{array}{l}\text { Comprou bebida alcoólica no posto de } \\
\text { gasolina }\end{array}$ & $21(65,6)$ & $22(73,3)$ & $43(69,4)$ & $0,69(0,23-2,06)$ \\
\hline Fumou maconha (últimas 24 horas) & $7(21,9)$ & $1(3,3)$ & $8(12,9)$ & $8,12(0,93-70,58)$ \\
\hline Fumou crack (últimas 24 horas) & $2(6,3)$ & $0(0)$ & $2(3,2)$ & - \\
\hline Cheirou cocaína (últimas 24 horas) & $3(9,4)$ & $0(0)$ & $3(4,8)$ & - \\
\hline Pretendia dirigir nas próximas 2 horas & $15(46,9)$ & $5(16,7)$ & $20(32,3)$ & $4,41(1,35-14,29)^{\ddagger}$ \\
\hline Acidente prévio após beber & $7(21,9)$ & $2(6,7)$ & $9(14,5)$ & $3,92(0,79-20,65)$ \\
\hline $\begin{array}{l}\text { Acidente prévio como passageiro de } \\
\text { motorista intoxicado }\end{array}$ & $8(25,0)$ & $6(20,0)$ & $14(22,6)$ & $1,33(0,42-4,23)$ \\
\hline Alcoolemia $>0,06$ e intenção de dirigir & $6(18,75)$ & $0(0)$ & $6(9,7)$ & - \\
\hline
\end{tabular}

IC95\% = intervalo de confiança de $95 \%$; OR = odds ratio.

* Mediana (mínino-máximo).

${ }^{\dagger}$ Tamanho do efeito.

$\$ \mathrm{p}<0,05$.

Qui-quadrado para variáveis categóricas/teste U de Mann-Whitney para variável assimétrica.

\section{Discussão}

Dados sobre comportamentos de risco relacionados a acidentes de trânsito são limitados no Brasil, e não há estudos publicados entre jovens que bebem em postos de gasolina - onde a combinação entre álcool e direção é quase inevitável. É importante observar que neste estudo foram entrevistados somente indivíduos que estavam obviamente ingerindo bebidas alcoólicas. Em média, esses jovens apresentavam 22 anos, faixa etária onde se encontram as mais altas taxas de óbito relacionadas a acidentes de trânsito no Brasil ${ }^{1}$. Além disso, a maioria dos indivíduos da amostra era estudante universitário, onde tipicamente se encontram altas 
prevalências de consumo excessivo de álcool (binge) e dirigir alcoolizado ${ }^{16}$.

Mesmo neste estudo piloto, a prevalência de alcoolemia acima do limite permitido por lei foi alarmante. Especificamente, quase metade dos homens apresentaram alcoolemia acima do limite legal, e mais de $1 / 3$ daqueles com alcoolemia ilegal pretendia dirigir nas 2 horas subseqüentes à entrevista, o que representa uma chance de 7 a 570 maior de ser morto em um acidente com um único veículo ${ }^{4}$. Essas prevalências estão de acordo com dados recentes de Duailib et al., que encontraram taxas de alcoolemia acima do limite legal em 19,4\% dos motoristas de Diadema ${ }^{17}$. O uso de maconha - que aumenta o risco de acidentes de trânsito ${ }^{18,19}$ - também foi muito alto e possivelmente subrelatado, uma vez que é ilegal.

$\mathrm{O}$ fato de nenhum dos motoristas ter aceitado a oferta de um táxi pode significar que os indivíduos desta amostra apresentavam baixa percepção de risco para beber e dirigir, sendo que esta certamente estava reduzida devido ao nível de álcool no sangue. Os motivos de saúde pública que podem justificar a recusa dessa medida preventiva possivelmente são a ausência de fiscalização de leis e a atitude tolerante da sociedade brasileira em relação ao consumo de álcool - que é incentivada por pesadas campanhas publicitárias ${ }^{20}$. Esses aspectos também auxiliam na compreensão do alto número de adolescentes abaixo de 18 anos que compraram bebidas alcoólicas nas lojas de conveniência. Nesse sentido, o estudo demonstrou a necessidade da discussão, com o Comitê de Ética em Pesquisa, sobre os procedimentos a serem seguidos ao abordar indivíduos abaixo da idade legal para comprar bebidas, uma vez que não houve um procedimento padrão para a situação.

Os resultados deste piloto demonstram que este tipo de estudo é viável no Brasil. A taxa de recusa foi baixa, especialmente considerando que muitos indivíduos apresentavam alcoolemia acima do limite permitido por lei e pretendiam dirigir. Essa baixa taxa de recusa pode ser explicada pela abordagem informal dos sujeitos de pesquisa, bem como pela utilização de linguagem semelhante à dos jovens. Os entrevistadores eram educados e treinados para desenvolver afinidade nas entrevistas clínicas, tinham idade semelhante e eram do mesmo estrato social dos entrevistados. O contato prévio com os proprietários dos postos de gasolina também facilitava a coleta de dados, pois a equipe já era reconhecida pelos seguranças dos postos antes de iniciar as entrevistas.

Existem limitações neste estudo que devem ser consideradas no futuro: 1) pequeno tamanho amostral; 2) amostra em alvo; 3 ) medidas auto-aplicáveis, exceto pela alcoolemia. Sugere-se a realização de estudos adicionais para examinar o consumo de álcool por menores de idade e para desenvolver intervenções direcionadas a esta população específica.
Este estudo gera pontos de referência para um conjunto maior de projetos que objetivem avaliar o uso de substâncias em motoristas que consomem bebidas alcoólicas em bares, restaurantes e estacionamentos pagos em Porto Alegre e outras cidades brasileiras, já contando com o auxílio financeiro do governo brasileiro. Isso deverá aumentar a validade externa dos dados e gerar manuais de treinamento para uso posterior.

\section{Referências}

1. Peden M, Scurfield R, Sleet D, Mohan D, Hyder AA, Jarawan E, et al. World report on traffic injury prevention. Geneva: WHO; 2004.

2. Ameratunga S, Hijar M, Norton R. Road-traffic injuries: confronting disparities to address a global-health problem. Lancet. 2006;367(9521):1533-40.

3. Connor J, Norton R, Ameratunga S, Jackson R. The contribution of alcohol to serious car crash injuries. Epidemiology. 2004;15(3):337-44.

4. Hingson R, Winter M. Epidemiology and consequences of drinking and driving. Alcohol Res Health. 2003;27(1):63-78.

5. Lardelli-Claret P, Jimenez-Moleon JJ, Luna-Del-Castillo Jde D, Bueno-Cavanillas A. Individual factors affecting the risk of death for rear-seated passengers in road crashes. Accid Anal Prev. 2006;38(3):563-6.

6. Duncan DF. Chronic drinking, binge drinking and drunk driving. Psychol Rep. 1997;80(2):681-2.

7. Cherpitel CJ. Prediction of alcohol-related casualties among emergency room admissions. Int J Addict. 1989;24(8):725-37.

8. Brasil, Ministério da Saúde, Secretaria de Vigilância em Saúde. Taxas padronizadas de óbitos por acidentes por transporte terrestre. Região e Brasil - 1996 a 2004. [citado 2006 Jul]. Disponível em: http://portal.saude.gov.br/saude/visualizar_texto.cfm?idtxt=24448.

9. Rodrigues CS, Ladeira RM, Pereira JC, Paula J, editores. Saúde em trânsito: pesquisa de acompanhamento de vítimas de acidentes de trânsito em Belo Horizonte. Belo Horizonte: Prefeitura de Belo Horizonte; 2005.

10. Nery Filho A, Medina MG, Melcop AG, Oliveira EM, editores. Impacto do uso de álcool e outras drogas em vitimas de acidentes de trânsito. Brasília: ABDETRAN, Instituto Raid, CETAD; 1997.

11. Galduróz JCF, Caetano R. Epidemiologia do uso de álcool no Brasil. Rev Bras Psiquiatr. 2004;26(Supl 1):3-6.

12. Galduroz JC, Noto AR, Nappo SA, Carlini EA. Household survey on drug abuse in Brazil: study involving the 107 major cities of the country-2001. Addict Behav. 2005;30(3):545-56.

13. Porto Alegre, Empresa Pública de Transporte e Circulação - EPTC. Dados gerais de acidentes de trânsito em Porto Alegre. [citado 2006 Jul]. Disponível em: http://www.eptc.com.br/Acidentes.asp.

14. Kypri K, Stephenson S. Drink-driving and perceptions of legally permissible alcohol use. Traffic Injury Prevention. 2005;6(3):219-24.

15. Blows S, Ivers RQ, Connor J, Ameratunga S, Woodward M, Norton R. Marijuana use and car crash injury. Addiction. 2005;100(5):605-11.

16. Hingson RW, Assailly JP, Williams AF. Underage drinking: frequency, consequences, and interventions. Traffic Inj Prev. 2004;5(3):228-36.

17. Duailibi S, Pinsky I, Laranjeira R. Prevalência do beber e dirigir em Diadema, estado de São Paulo. Rev Saude Publica. 2007;41(6):1058-61.

18. Kelly E, Darke S, Ross J. A review of drug use and driving: epidemiology, impairment, risk factors and risk perceptions. Drug an Alcohol Rev. 2004;23(3):319-44.

19. Fergusson DM, Horwood LJ. Cannabis use and traffic accidents in a birth cohort of young adults. Accid Anal Prev. 2001;33(6):703-11.

20. Pinsky I, Pavarino Filho RV. A apologia do consumo de bebidas alcoólicas e da velocidade no trânsito no Brasil: considerações sobre a propaganda de dois problemas de saúde pública. Rev Psiquiat RS. 2007;29(1):110-8. 